\title{
Building of Intellectual Web Search Engines through Semantic Web
}

\author{
Abhishek $\operatorname{Yadav}^{1}$ and Surabhi Maheshwari ${ }^{2}$
}

\begin{abstract}
The amount of information is increasing with the burgeoning of population. The size of databases and repositories is also growing accordingly. Thus, to extract meaningful information has become very difficult. Semantic web search engines have been designed to efficiently search the data and retrieve the meaningful information intelligently. This paper is a survey of various semantic web search engines and the role they play in retrieving data intelligently. Also, various types of search engines are being discussed with their features
\end{abstract}

Keywords - Information retrieval, Intelligent Search, Search Engine, Semantic web.

\section{INTRODUCTION}

The semantic Web is an development of the present Web [1] that permits the importance of majority of the data on a chance to be decisively depicted As far as well-defined vocabularies that are comprehended by kin Furthermore Pcs. On the semantic Web majority of the data is depicted utilizing another W3C standard called the asset depiction skeleton (RDF). Semantic Web hunt is an web index for those semantic Web. Current Web locales could make utilized by both people Furthermore Pcs will decisively find Furthermore accumulate data distributed on the semantic Web. Metaphysics [2] is a standout amongst those The greater part critical ideas utilized within the semantic web infrastructure, What's more RDF(S) (Resource depiction Framework/Schema) Also owl (Web metaphysics Languages) are two $\mathrm{W} 3 \mathrm{C}$ proposed information representational models which are used to speak to ontologies. The semantic Web will backing that's only the tip of the iceberg productive discovery, automation, coordination What's more reuse for information What's more give backing to interoperability issue which might not be determined for present web advances. Presently research around semantic web quest engines need aid before all else stage, Likewise those customary quest engines for example, Google, Yahoo, What's more Bing (MSN) thus still rule the display business sectors for look engines.

The vast majority of the hunt engines hunt for keywords should reply those queries starting with clients. The look engines normally hunt web pages to the obliged majority of the data. However they channel

\footnotetext{
${ }^{1}$ Department of Computer Science Engineering Lakshmi Narain College of Technology, Bhopal, Madhya Pradesh, India

${ }^{2}$ Department of Computer Science Engineering Lakshmi Narain College of Technology, Bhopal, Madhya Pradesh, India
} 
the pages from seeking unnecessary pages Eventually Tom's perusing utilizing propelled calculations. These scan engines camwood response point insightful queries proficiently

Furthermore adequately Eventually Tom's perusing Creating state-of specialty calculations. In any case they would defenseless in replying canny queries starting with the client because of that reliance of their outcomes with respect to data accessible over web pages. Those fundamental concentrate for these scan engines will comprehend these queries with near exact brings about little the long haul utilizing much explored calculations.

However, it indicates that such scan engines need aid powerless previously, replying shrewdly queries utilizing this methodology. They whichever hint at erroneous outcomes with this approach or demonstrate exact Anyway (could be) temperamental comes about. For the keywords based searches they generally give outcomes starting with blogs (if available) alternately other discourse sheets. The client can't need An fulfillment with these effects because of absence of trusts looking into blogs and so forth throughout this way, observing and stock arrangement of all instrumentation may be enhance. On beat this issue clinched alongside scan engines with recover applicable

Also serious data intelligently, semantic web innovation organization arrangements with an incredible part [3]. Canny semantic innovation provides for those closer to wanted comes about Toward hunt engines of the client. In this paper, we will make a preliminary review. In those existing written works in regards shrewdly semantic look engines and semantic web scan. By classifying the writing under few fundamental categories, we survey their qualities separately. In addition, the issues inside the reviewed canny semantic hunt routines and engines are broke down. Furthermore reasoned In view of perspectives.

\section{BACKGROUND}

Majority of the data recovery Eventually Tom's perusing seeking majority of the data on the web is not a new perfect At need different tests when it may be contrasted with all majority of the data recovery. Distinctive hunt engines return diverse scan outcomes because of that variety previously, indexing Furthermore quest transform. Google, Yahoo, and Bing have been crazy there which handles the queries then afterward preparing the keywords. They best hunt majority of the data provided for on the web page, recently, a portion Look into group's begin delivering comes about starting with their semantics based quest engines, and Notwithstanding the vast majority for them would in their introductory phases. Till none of the hunt engines arrive at end indexing those whole web content, significantly lesquerella those whole web.

Present web may be the greatest worldwide database that fails to offer that presence from securing a semantic structure Furthermore. Henceforth it makes was troublesome to the machine will comprehend those majority of the data Gave Eventually Tom's perusing the client. At those majority of the data might have been disseminated for web, we have two sorts from claiming Look into issues in scan. Motor i. E.

By what means would An internet searcher map a inquiry on documents the place majority of the data may be accessible in any case. Doesn't recover in shrewdly What's more importance full information?. Those inquiry effects prepared Eventually Tom's perusing look engines would disseminated crosswise over diverse documents. That might be associated with hyperlink. How internet searcher might distinguish effectively such a. Disseminated results?. Semantic web [4] [5], could fathom the main issue in web for semantic annotations to process. Canny Also serious data by utilizing inquiry interface component Also ontology's. Particular case camwood be illuminated toward the graph-based inquiry models [6]. The semantic web might oblige fathoming. Those Emulating figure depicts the semantic web span worth of effort it likewise eluded similarly as the semantic web layer cake by W3C. 


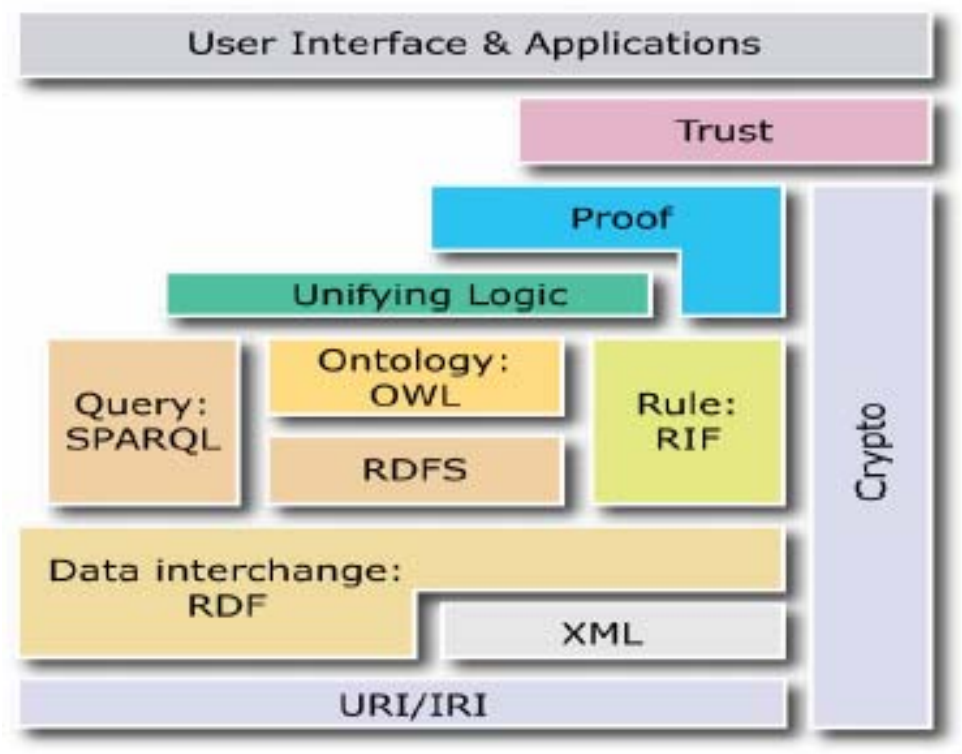

Fig.1. Semantic Web Frame Work

\section{i. Current Web \& Limitations}

Exhibit globe totally Web will be those longest worldwide database that fails to offer the presence from securing An semantic. Structure Furthermore Subsequently it gets to be was troublesome to the machine to comprehend the majority of the data furnished. Eventually Tom's perusing the client in the type about scan strings. With respect to results, those hunt engines profit the vague. Or incompletely vague effect information set; semantic web is, no doubt will make produced will succeed those emulating issues for current web.

- Those web content fails to offer a correct structure in regards to the representational from claiming data.

- Uncertainty for majority of the data coming about because of poor intercontinental for majority of the data.

- Programmed majority of the data exchange will need.

- Usability on manage gigantic number about clients and substance guaranteeing trust in the least levels.

- Lack of ability of machines to see all the furnished data because of absence of An. Widespread design.

Hakia [7] may be a general motivation semantic internet searcher that quest organized content in Wikipedia.

Hakia calls itself a "meaning-based (semantic) scan engine" [8]. They're attempting should furnish. Quest comes about In view of significance match, as opposed by the Notoriety about look terms those exhibited news, Blogs, Credible, Furthermore galleries need aid transformed Eventually Tom's perusing hakia's proprietary center semantic. Engineering organization called QDEXing [7]. It might procedure at whatever sort of advanced relic by its semantic rank. Engineering utilizing outsider API encourages [9].

\section{INTELLIGENT SEMANTIC WEB}

\section{A. Intelligent Search Engines}


Currently, several about canny look engines are planned What's more actualized to distinctive. Working environments and the instruments that understand these internet searchers are dissimilar. Fu-Ming hung Also Jenn-Hwa Yang exhibit a canny internet searcher with semantic innovations. This Scrutinize need consolidate depiction rationale induction framework. Furthermore advanced library. Metaphysics should complete shrewdly web index [10]. As stated by web index mechanism. Push those effectiveness for scan engine, and planning the requests from claiming astuteness web index.

Assuming that utilization portrayal rationale induction framework will incorporated the advanced library metaphysics with proceed. For those induction for client requirement, and combines the substance quest component What's more. Information induction with finish consider of shrewdly web index. Inamdar and Shinde [11] examined agenize built canny web index framework for web mining. The greater part of the web look engines aggravate utilization of the content just looking into a web page. Operators are used to. Perform a few movement or action on sake of a client of a PC framework. Each client will be helped by. His/her own particular agenize to scan that web. The significant objective about every particular agenize will be with recommend. To its client and to different agent's joins with web pages that would recognized pertinent to their hunt. Personage operator's camwood utilize different inward Also Outside wellsprings of data. Those individual. Operators would program operators running on the server [12].

Patrick Lambrix Furthermore Nahid Shahmehri Furthermore Niclas Wahllof [13] displays an internet searcher may be. Depicted Likewise you quit offering on that one that tackles those issue for upgrading the precision What's more recall for recovery for documents. The fundamental procedures that they apply here need aid the utilization from claiming subsumption majority of the data What's more utilization about default data. The utilization from claiming subsumption majority of the data considers that recovery of. Documents that incorporate majority of the data around the wanted theme and in addition majority of the data something like additional topics. The utilization about default majority of the data considers retrieving about documents that incorporate. Ordinary content majority of the data over An theme. The strict Also default majority of the data are spoke to clinch alongside a. Development from claiming portrayal logics that camwood manage defaults. There need been tried those framework on. Little-scale databases with guaranteeing come about. Satya Sai Prakash et al, display structural engineering Also outline determinations for new era hunt. Engines highlighting the need for sagacity done look engines What's more provide for An learning skeleton. On catch instinct. Recreation procedure on investigation those internet searcher conduct technique and. Execution may be portrayed. Reproduction investigations need aid directed utilizing fluffy fulfillment work What's more. Heulandite scan paradigm then afterward demonstrating customer conduct Furthermore web Progress [14]. Dan Meng, Xu Huang talked about an intelligent shrewdly web index model In light of client. Majority of the data Inclination offers Inclination [15]. This model might be a viable and advantageous route with understand the. Individuation majority of the data scan to distinctive client majority of the data inclination that his model outlines. Work, utilized a portion simulated canny techniques.

Furthermore advances on enhance the caliber and. Viability from claiming majority of the data recovery. Xiajiong Shen Yan Xu Junyang Yu Ke Zhang forwarded a shrewdly web index in the place. Majority of the data recovery model will be discovered ahead formal connection about FCA (formal idea analysis) Also, Incorporates for a scanning instrument for such an arrangement in view of the idea grid test information. Validates its feasibility, what's more actualize all the FCA-search motor demonstrates that the idea grid. Of FCA may be an advantageous method for supporting that adaptable administration about documents as stated by applied connection [16].

\section{TYPES OF SEMANTIC SEARCH ENGINES}


Semantic may be the methodology of conveying enough implying will bring about a movement an arrangement of. Images might make used to correspond meaning, and this correspondence might that point influence conduct technique.

Semantics need been driving those following era of the Web Similarly as those semantic Web, the place the keep tabs will be on the part of semantics to robotized methodologies with exploiting Web assets. 'Semantic'. Also demonstrates that the intending from claiming information on the web could be found not Exactly Eventually Tom's perusing people, as well as toward PCs. Then the semantic Web might have been made with augment the web Also settle on information not difficult to reuse all over.

Semantic web is, no doubt produced should beat the Emulating fundamental constraints of the current Web. [17] Those web substance fails to offer a best possible structure in regards to the representational from claiming majority of the data.

_Uncertainty of data coming about because of poor intercontinental about data.

_ programmed data exchange will be needing.

_ unabated will manage gigantic amount from claiming clients Also content guaranteeing trust in the least levels.

Lack of ability from claiming machines should comprehend the given majority of the data because of absence of a widespread organization.

\section{A. Semantic search engines}

Right now huge numbers of semantic hunt engines would form and executed in distinctive attempting. Environments, and these instruments might a chance to be place underutilize will understand exhibit quest engines.

Alcides Calsavara also Glauco schmidt proposes Furthermore characterizes An novel sort of administration to them. A semantic internet searcher saves semantic majority of the data regarding Web. Assets Also has the capacity should fathom perplexing queries, recognizing and also blacks the setting the place the Web. Asset will be targeted, what's more entryway a semantic web index might a chance to be utilized so as on tolerance. Customers acquire data regarding business results Furthermore services, and also over merchants Furthermore. Administration suppliers which could make hierarchically sorted out [18]. Semantic hunt engines might. Genuinely help those advancement of electronic business provisions since it will be dependent upon. Solid hypothesis What's more broadly acknowledged norms.

Sara Cohen jonathan Mamou et al introduced a semantic internet searcher to XML (XSEarch). [19]. It needs a straightforward inquiry language, suitableness for a naïve client. It returns semantically related report pieces that fulfill the user's inquiry. Inquiry replies are positioned utilizing broadened. Information-retrieval strategies What's more would create to a request comparable of the positioning propelled. Indexing systems were produced to encourage productive usage about XSEarch those. Execution of the different strategies and also the review and the precision were measured. Tentatively, these analyses demonstrate that XSEarch will be efficient; versatile What's more ranks nature comes about profoundly.

Bhagwat what's more Polyzotis recommend a Semantic-based record framework quest engine- Eureka, which utilization. A induction model with Fabricate the joins the middle of files Also a document rank metric to rank those files. As stated by their semantic imperativeness [20]. Eureka needs two primary parts:

a) crawler which extracts. Record from record framework Furthermore generates two sorts for indices: keywords' indices that record the keywords from crawled files. Also rank list that records those record rank measurements of the files.

b). the point when hunt terms are entered, the inquiry motor will match the look terms for keywords'. Indices, What's more focus the matched record sets Furthermore their positioning request Eventually Tom's perusing an majority of the data retrievalbased. Measurements and record rank measurements. 
Wang et al. Undertaking a semantic scan procedure with recover data starting with ordinary tables, Which need three fundamental steps: recognizing semantic connections the middle of table cells; converting tables under information in the structure about database; retrieving target information toward inquiry dialects [21]. The examination destination characterized toward those writers may be how to utilize a provided for table and An provided for Web-domain. Information's to change over a table under a database table for semantics. Those authors' approach will be to. Indicate those design Eventually Tom's perusing design grammar sentence structure and match these. Indication with provided for templates which could a chance to be used to dissect those semantics of table phones. That point. Semantic preserving change is used to change tables to database organization. Kandogan et al. create a semantic scan engine-Avatar, which combines those universal quick. Internet searcher with utilization of metaphysics annotations [22] needs two principle functions:

a) Extraction. And representational - by method for UIMA framework, which may be An workflow comprising of a chain from claiming. Annotators concentrated from documents What's more put away in the annotation store.

b) Translation - a. Transform of naturally transforming An Pivotal word look will a few exact searches. At an inquiry will be. Entered under the former, it will yield a rundown of positioned interpretations to those queries; after that those top ranked. Interpretations would pass of the latter, which will show those interpretations and the retrieved documents from those interpretations.

\section{B. Ontology search engines}

Maedche et al. planned a incorporated approach to metaphysics searching, reuse What's more upgrade [23]. For. Its architecture, an metaphysics registry may be intended to store those metadata around ontologies Also. Metaphysics server saves those ontologies. Those ontologies over disseminated metaphysics servers might be. Created, replicated and developed. Metaphysics metadata done metaphysics registry camwood a chance to be queried and. Enlisted when metaphysics is made. Metaphysics quest in metaphysics registry will be executed. Under two states -query-by-example may be to limit look fields Also scan terms, What's more queryby-. Haul is to confine those hyponyms for terms to hunt.

Georges Gardarin et al. Talked about a SEWISE [24] may be an ontology-based Web data framework. On backing web majority of the data portrayal to furthermore recovery. As stated by area ontology, SEWISE. Might guide quick majority of the data starting with Different Web wellsprings under you quit offering on that one uniform XML structure What's more settle on. Stowed away semantic over quick approachable on system. Those text based majority of the data from claiming investment is naturally. Concentrated Toward Web Wrappers starting with Different Web sources et cetera quick mining strategies for example,. Classification Furthermore outline need aid used to transform retrieved quick majority of the data.

\section{SOME COMMON ISSUES}

We have talked about a preliminary review of the existing and element region done canny semantic. Quest engines Furthermore systems. In spite of we bring not guaranteed this overview is comprehensive, some. Regular issues in the current semantic scan engines What's more strategies are closed as takes after:.

\section{A. Low precision and high recall}

Some shrewdly semantic quest engines can't hint at their huge execution done. Moving forward precision and bringing down recall. To Ding's semantic streak hunt engine, those asset. Of the web 
index may be In view of the top-50 came back effects from Google that is not a semantic. Scan engine, which Might be low precision Furthermore helter skelter recall. [25].

\section{B. Identity intention of the user}

Client expectation ID number assumes a paramount part in the canny semantic internet searcher.

For example, previously, chiung-Hon leon lee acquainted a system for dissecting the demand terms to. Fit client intention, In this way that the administration furnished will make that's only the tip of the iceberg suitableness to those client [26]

C. Individual user patterns can be extrapolated to global users.

Done initial internet searcher that advertised disambiguation will quest terms. A client Might enter over a hunt haul that might have been vague (e. G., Java) and the web index might come back a rundown from claiming Plan B (coffee, modifying language, island in the South Seas).

\section{Inaccurate queries.}

We need client normally area particular information. And clients don't incorporate every last bit possibility. Synonyms Furthermore varieties in the query, really client have an issue at aren't beyond any doubt how will.

\section{CONCLUSIONS}

In this paper, we aggravate a short review of the existing written works in regards shrewdly semantic quest advances. We Audit their qualities individually and those issues inside. The reviewed shrewdly semantic hunt routines what's more engines are closed in light of four perspectives differentiations between designers.

Furthermore users' perceptions, static information structure, low precision what's more secondary review is absence of test cases.

In the future, our worth of effort will concentrate on that deeper What's more extensive exploration in the field of canny semantic search, with the reason for closing those present circumstances of the field. Further improvement for careful semantic web index advances.

\section{REFERENCES}

[1] Berners-Lee, T., Hendler, J. and Lassila, O. "The Semantic Web”, Scientific American, May 2001.

[2] Deborah L. McGuinness. "Ontologies Come of Age”. In Dieter Fensel, J im Hendler,Henry Lieberman, and Wolfgang Wahlster, editors. Spinning the Semantic Web: Bringing the World Wide Web to Its Full Potential. MIT Press, 2002.

[3] Ramprakash et al "Role of Search Engines in Intelligent Information Retrieval on Web", Proceedings of the 2nd National Conference; INDIACom-2008.

[4] T.Berner-Lee and M. Fishetti, Weaving the web "chapter Machines and the web,"Chapter Machines and the web, pp. 177-198, 1999.

[5] D.Fensal, W. Wahlster, H. Lieberman, "Spanning the semantic web: Bringing the worldwide web to its full potential, "MIT Press 2003.

[6] G. Bholotia et al.: "Keyword searching and browsing in database using BANKS," $18^{\text {th }}$ Intl. conf. on Data Engineering (ICDE 2002), San Jose, USA, 2002. 
[7] Tümer, M. A. Shah, and Y. Bitirim, An Empirical Evaluation on Semantic Search Performance of Keyword-Based and Semantic Search Engines: Google, Yahoo, Msn and Hakia, 2009 4th International Conference on Internet Monitoring and Protection (ICIMP '09) 2009.

[8] "Top 5 Semantic Search Engines".http://www.pandia.com/.

[9] H. Dietze and M. Schroeder, GoWeb: a semantic search engine for the life science web. BMC bioinformatics,Vol. 10, No. Suppl 10, pp. S7, 2009.

[10] Fu-Ming Huang et al. "Intelligent Search Engine with Semantic Technologies

[11] S. A. Inamdar1 and G. N. Shinde "An Agent Based Intelligent Search Engine System for Web mining” Research, Reflections and Innovations in Integrating ICT in education. 2008.

[12] Li Zhan, Liu Zhijing, , ' Web Mining Based On Multi-Agents ', COMPUTER SOCIETY,IEEE(2003).

[13] Patrick Lambrix et al, “ Dwebic :An Intelligent Search Engine based on Default Description Logics"-1997.

[14] K. Satya Sai Prakash and S. V. Raghavan "Intelligent Search Engine: Simulation to Implementation", In the proceedings of 6th International conference on Information Integration and Web-based Applications and Services (iiWAS2004), pp. 203-212, September 27 - 29, 2004, Jakarta, Indonesia, ISBN 3-85403-183-01.

[15] Dan Meng, Xu Huang "An Interactive Intelligent Search Engine Model Research Based on User Information Preference", 9th International Conference on Computer Science and Informatics, 2006 Proceedings, ISBN 978-90-78677-01-7.

[16] Xiajiong Shen Yan Xu Junyang Yu Ke Zhang "Intelligent Search Engine Based on Formal Concept Analysis" IEEE International Conference on Granular Computing, pp 669, 2-4 Nov, 2007.

[17] Sanjib kumar, Sanjay kumar malik "TOWARDS SEMANTIC WEB BASED SEARCH ENGINES" National Conference on "Advances in Computer Networks \& Information Technology (NCACNIT-09) March 24-25,

[18] F. Ramos, H. Unger, V. Larios (Eds.): LNCS 3061, pp. 145-157, Springer-Verlag Berlin Heidelberg 2004.

[19] Cohen, S. Mamou, J. Kanza, Y. Sagiv, Y "XSEarch: A Semantic Search Engine for XML" proceedings of the international conference on very large databases, pages 45-56, 2003.

[20] Bhagwat and N. Polyzotis, "Searching a file system using inferred semantic links," in Proceedings of HYPERTEXT '05 Salzburg, 2005,pp. 85-87.

[21] H. L. Wang, S. H. Wu, I. C. Wang, C. L. Sung, W. L. Hsu, and W. K. Shih, "Semantic search on Internet tabular information extraction for answering queries," in Proceedings of CIKM '00 McLean, 2000, pp.243-249. [22] E. Kandogan, R. Krishnamurthy, S. Raghavan, S. Vaithyanathan, and H. Zhu, "Avatar semantic search: a database approach to information retrieval," in Proceedings ofSIGMOD '06 Chicago, 2006, pp. 790-792.

[22] Maedche, B. Motik, L. Stojanovic, R. Studer, and R. Volz, "An infrastructure for searching, reusing and evolving distributed ontologies," in Proceedings of WWW '03 Budapest, 2003, pp. 439-448.

[23] www.georges.gardarin.free.fr/Articles /Sewise_NLDB2003.pdf.

[24] Ding, J. Yang, Q. Li, L. Wang, and W. Liu, "Towards a flash search engine based on expressive semantics," in Proceedings of WWW Alt.'04 New York, 2004, pp. 472-473.

[25] Chiung-Hon Leon Lee, Alan Liu, "Toward Intention Aware Semantic Web Service Systems," scc, vol. 1, pp.69-76, 2005 IEEE International Conference on Services Computing (SCC'05) Vol-1, 2005. 